\title{
Holographic characterization of protein aggregates
}

Chen Wang ${ }^{1}$, Xiao Zhong ${ }^{2}$, David B. Ruffner ${ }^{3}$, Alexandra Stutt ${ }^{4}$, Laura Philips ${ }^{3}$, Michael D. Ward ${ }^{2}$, and David G. Grier ${ }^{1, *}$

${ }^{1}$ Department of Physics and Center for Soft Matter Research, New York University, New York, NY 10003

${ }^{2}$ Department of Chemistry and Molecular Design Institute, New York University, New York, NY 10003

${ }^{3}$ Spheryx, Inc., 330 E. 38th Street, New York, NY 10016

${ }^{4}$ Packer Collegiate Institute, 170 Joralemon Street, Brooklyn, NY 11201

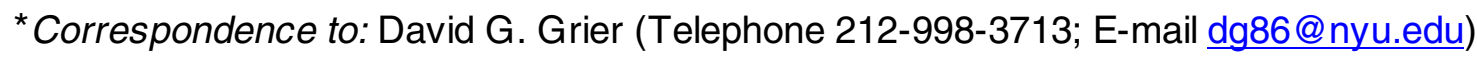

Keywords: Biopharmaceuticals characterization, Protein aggregation, Particle size, Light scattering, Microscopy, Microparticles, Colloids 


\section{Abstract}

We demonstrate how holographic video microscopy can be used to detect, count and characterize individual micrometer-scale protein aggregates as they flow down a microfluidic channel in their native buffer. Holographic characterization directly measures the radius and refractive index of subvisible protein aggregates, and offers insights into their morphologies. The measurement proceeds fast enough to build up population averages for time-resolved studies, and lends itself to tracking trends in protein aggregation arising from changing environmental factors. Information on individual particles' refractive indexes can be used to differentiate protein aggregates from such contaminants as silicone droplets. These capabilities are demonstrated through measurements on samples of bovine pancreas insulin aggregated through centrifugation and of bovine serum albumin aggregated by complexation with a polyelectrolyte. Differentiation is demonstrated with samples that have been spiked with separately characterized silicone spheres. Holographic characterization measurements are compared with results obtained with micro-flow imaging and dynamic light scattering. 


\section{INTRODUCTION}

The tendency of proteins to aggregate into clusters is a major concern in manufacturing protein-based biopharmaceuticals and assessing their safety. Beyond their reduced therapeutic efficacy, protein aggregates elicit immune responses that result in clinical adverse events ${ }^{1-6}$. Detecting, counting and characterizing protein aggregates is essential to understanding the critical pathways responsible for protein aggregation. Established particle characterization technologies such as dynamic light scattering (DLS) work well for in situ characterization of sub-micrometer-scale aggregates, but are less effective for larger subvisible aggregates ${ }^{7,8}$. Other techniques, such as micro-flow imaging $(\mathrm{MFI})^{9,10}$, are better suited for aggregates larger than a micrometer or $\mathrm{so}^{8,10}$, but do not provide information on composition. Comparatively few established techniques probe the size and composition of protein aggregates in the subvisible range from $100 \mathrm{~nm}$ to $10 \mathrm{~m}^{8}$. The need for enhanced characterization techniques is particularly acute in applications that require real-time monitoring of subvisible aggregates in their native environment.

Herein we introduce an innovative method based on holographic video microscopy ${ }^{11}$ for detecting, counting and characterizing individual colloidal particles in suspension. This technique naturally distinguishes protein aggregates from contaminants such as silicone droplets based on differences in their optical properties. Holographic characterization rapidly builds up population statistics on subvisible protein aggregates in their natural state, without requiring dilution or special solvent conditions. Its precision, accuracy and range of operation are demonstrated through measurements on model suspensions of colloidal spheres. Its utility for characterizing 
protein aggregates is illustrated through measurements on aggregates grown in solutions of bovine pancreas insulin and bovine serum albumin, including measurements on samples deliberately spiked with silicone spheres. Comparisons with micro-flow imaging and dynamic light scattering highlight strengths and weaknesses of holographic characterization for analyzing subvisible protein aggregates in the micrometer size range. This study concludes by assessing how holographic characterization complements established particle-characterization techniques for analyzing aggregation in protein formulations.

\section{METHODS AND MATERIALS}

\section{Holographic Characterization}

Our measurement technique, shown schematically in Figure 1, is based on in-line holographic video microscopy ${ }^{11-13}$, a technique that creates holograms of individual objects in the microscope's field of view. A holographic microscope illuminates its sample with a collimated laser beam rather than a conventional incoherent light source. Light scattered by an aggregate therefore interferes with the remainder of the beam in the focal plane of an optical microscope. The microscope magnifies this interference pattern and projects it onto the face of a video camera, which records the spatially varying intensity pattern, $I(\mathbf{r})$. Each image in the resulting video stream is a holographic snapshot of the scatterers passing through the laser beam, and can be analyzed with predictions ${ }^{11,14,15}$ based on the Lorenz-Mie theory of light scattering ${ }^{16-}$ 18 to measure each aggregate's radius, $a_{p}$, refractive index, $n_{p}$, and three-dimensional position, $\mathbf{r}_{p}^{11}$.

Holographic characterization originally was developed for analyzing spherical 
particles ${ }^{11,19}$. Rigorously generalizing the analysis to account for the detailed structure of aspherical and inhomogeneous objects is prohibitively slow because of the analytical complexity and the associated computational burden ${ }^{20-22}$. We therefore use the idealized spherical model to characterize protein aggregates with the understanding that the results should be interpreted as referring to an effective sphere comprising both the protein aggregate and the fluid medium that fills out the effective sphere. We previously have demonstrated that this approach yields reliable results for porous colloidal particles ${ }^{23}$ and for dimpled spheres ${ }^{24}$. The goal of the present study is to demonstrate its utility for subvisible protein aggregates.

\section{Holographic video microscope}

Our custom-built holographic microscope uses a standard microscope objective lens (Nikon Plan Apo, 100x, numerical aperture 1.45, oil immersion) in combination with a tube lens to attain a system magnification of $135 \mathrm{~nm} / \mathrm{pixel}$ on the face of a monochrome video camera (NEC Tl-324All). We illuminate the sample with the $1 \mathrm{~mm}$ diameter collimated beam from a solid state laser (Coherent DPSS), which delivers 10 $\mathrm{mW}$ of light to the sample at a vacuum wavelength of $532 \mathrm{~nm}$ and a peak irradiance of $10 \mathrm{~mW} / \mathrm{mm}^{2}$. For micrometer-scale colloidal spheres, this instrument can measure an individual particle's radius with nanometer precision ${ }^{19,25}$, its refractive index to within a part per thousand 25,26 , and can track its position to within a nanometer in the plane and to within 3 nanometers along the optical axis ${ }^{14,15,25}$. Each fit can be performed in a few tens of milliseconds using automated feature detection ${ }^{27}$ and image recognition algorithms ${ }^{28}$. A single fit suffices to characterize a single protein aggregate.

\section{Holographic characterization procedure}


To characterize the population of aggregates in a protein dispersion, we flow the sample through the microscope's observation volume in a microfluidic channel ${ }^{19}$. Given the camera's exposure time of $0.1 \mathrm{~ms}$, results are immune to motion blurring for flow rates up to $100 \mathrm{~m} / \mathrm{s}^{19,29}$. Under typical conditions, no more than ten protein aggregates pass through the $86 \mathrm{~m} \times 65 \mathrm{~m}$ field of view at a time. These conditions simplify the holographic analysis by minimizing overlap between individual particles' scattering patterns. Each aggregate typically is recorded in multiple video frames as it moves through the field of view. Such sequences of measurements are linked into trajectories using a maximum-likelihood algorithm ${ }^{30}$ and median values are reported for each trajectory ${ }^{19}$. These considerations establish an upper limit to the range of accessible aggregate concentrations of $10^{8}$ aggregates $/ \mathrm{mL}$. At the other extreme, 10 min of data suffices to detect, count and characterize aggregates at concentrations as low as $10^{4}$ aggregates $/ \mathrm{mL}$.

The scatter plot inset into Figure 1 shows typical results for subvisible aggregates of bovine insulin. Each point represents the properties of a single aggregate, and is comparable in size to the estimated errors in the radius and refractive index ${ }^{11,15}$. Colors represent the local density $\left(a_{p}, n_{p}\right)$ of recorded data points in the $\left(a_{p}, n_{p}\right)$ plane, computed with a kernel density estimator ${ }^{31}$, with red indicating the most probable values.

\section{Calibrating the holographic characterization instrument}

Holographic characterization relies on four instrumental calibration parameters: the vacuum wavelength of the laser illumination, the magnification of the optical train, the dark count of the camera, and the single-pixel signal-to-noise ratio at the operating 
illumination level. All of these can be measured once and then used for all subsequent analyses.

The vacuum wavelength of the laser is specified by the manufacturer and is independently verified to four significant figures using a fiber spectrometer (Ocean Optics, HR4000). The microscope's system magnification is measured to four significant figures using a precision micrometer scale (Ted Pella, catalog number 2285-16). The camera's dark count is measured by blocking the laser illumination and computing the average image value at each pixel. Image noise is estimated from holographic images with the median-absolute-deviation (MAD) metric.

In addition to these instrumental calibrations, obtaining accurate results also requires an accurate value for the refractive index of the medium at the laser wavelength and at the sample temperature. For the aqueous buffers in the present study, this value was obtained to four significant figures with an Abbe refractometer (Edmund Optics). Approximating this value with the refractive index of pure water, $n_{m}=$ 1.335 , at the measurement temperature of $21 \pm 1 \mathrm{C}$ yields systematic errors in the estimated radius and refractive index of no more than $0.1 \%$.

Matching the refractive index of the medium to that of the protein suppresses light scattering by protein aggregates and thus reduces the contrast and the signal-to-noise ratio of recorded holograms. Holographic characterization is more effective for samples with larger index mismatch between the medium and the scattering particles. This limitation is shared by any non-fluorescent imaging technique.

\section{Operating range of holographic characterization}

The operating range of the holographic characterization instrument is 
established by measurements on aqueous dispersions of colloidal spheres intended for use as particle size standards. The interference fringes in each particle's holograms must be separated by at least one pixel in the microscope's focal plane. This requirement is accommodated by setting the focal plane $5 \mathrm{~m}$ below the glasswater interface in the sample cell. The largest accessible axial displacement is set both by the need to fit multiple concentric fringes into the camera's field of view, and also by the reduction of image contrast below the camera's noise floor. This upper limit is roughly $100 \mathrm{~m}$ for this instrument. In practice, however, we pass samples through microfluidic channels with an optical path length of $30 \mathrm{~m}$ to ensure good imaging conditions for all aggregates, regardless of their height in the channel.

The lower end of the range of detectable particle sizes is limited to half the wavelength of light in the medium. Particles smaller than this yield detectable holograms, which can be fit by Lorenz-Mie theory. These fits, however, do not cleanly separate the particle size from the refractive index. If the particle's refractive index is known a priori, these measurements again can yield reliable estimates for the particle's radius. For the present measurements, the practical lower limit is set by the 8-bit dynamic range of the camera to $a_{p} 200 \mathrm{~nm}$. Smaller particles' light-scattering patterns lack the contrast needed for reliable detection and characterization.

The upper size limit is set to $a_{p} 10 \mathrm{~m}$ by the depth of the channel. We do not, moreover, expect to observe and correctly identify irregularly shaped protein aggregates much larger than $10 \mathrm{~m}$. Highly asymmetric aggregates substantially larger than the wavelength of light are likely to be misidentified as two or more distinct features by the feature-identification algorithm developed for automated holographic 
characterization of spheres ${ }^{14,27}$. No effort is made to correct for this artifact in the present study, although its presence is confirmed by comparing results from holographic characterization with results obtained through micro-flow imaging analysis.

\section{Holographic morphology measurements and micro-flow imaging}

The same holograms used for holographic characterization through Lorenz-Mie analysis also can be used to visualize the three-dimensional morphology of individual aggregates through Rayleigh-Sommerfeld back-propagation ${ }^{13}$ with volumetric deconvolution ${ }^{32}$. This technique uses the Rayleigh-Sommerfeld diffraction integral to reconstruct the volumetric light field responsible for the observed intensity distribution. The object responsible for the scattering pattern appears in this reconstruction in the form of the caustics it creates in the light field ${ }^{13,14}$. For objects with features comparable in size to the wavelengths of light, these caustics have been shown to accurately track the position and orientation of those features in three dimensions ${ }^{33}$. Deconvolving the resulting volumetric data set with the point-spread function for the Rayleigh-Sommerfeld diffraction kernel eliminates twin-image artifacts and yields a three-dimensional representation of the scatterer ${ }^{32}$.

Volumetric reconstructions of protein aggregates can be projected into the imaging plane to obtain the equivalent of bright-field images in the plane of best focus. This reaps the benefit of holographic microscopy's very large effective depth of focus compared with conventional bright-field microscopy. The resulting images are useful for micro-flow imaging analysis, including analysis of protein aggregates' sizes and morphology. An aggregate's radius can be estimated from the projected reconstruction 
as the radius of gyration of the aggregate's image.

In addition to providing an alternative approach to measuring aggregate size, this holographic approach to micro-flow imaging can be used to assess the rate of false feature identifications in the Lorenz-Mie analysis, and thus the rate at which larger aggregates are misidentified as clusters of smaller aggregates. Investigating aggregate size and morphology with holographic deconvolution microscopy thus is a useful complement to holographic characterization through Lorenz-Mie analysis. Whereas Lorenz-Mie fits proceed in a matter of milliseconds, however, RayleighSommerfeld back-propagation is hundreds of times slower. The present study focuses, therefore, on the information that can be obtained rapidly through Lorenz-Mie characterization.

\section{Dynamic Light Scattering}

Dynamic light scattering measurements were performed with a Zetasizer Nano ZS (Malvern Instruments), which operates in the back-scattering geometry at a vacuum wavelength of $633 \mathrm{~nm}$. Samples for analysis were introduced into the disposable polystyrene cuvette (DTS0012, Malvern Instruments) and allowed to thermally equilibrate to $25 \mathrm{C}$ within the instrument for $5 \mathrm{~min}$. Each sample was scanned 10 times, and the average results for particle counts were tabulated.

\section{Preparation of Protein Samples}

\section{Bovine pancreas insulin}

Samples of bovine pancreas insulin (Mw: $5733.49 \mathrm{Da}$, Sigma-Aldrich, CAS number: $11070-73-8)$ were prepared according to previously published methods ${ }^{34,35}$ with modifications for investigating insulin aggregation induced by agitation alone. 
Insulin was dissolved at a concentration of $5 \mathrm{mg} / \mathrm{mL}$ in $10 \mathrm{mM}$ Tris buffer (Life Technologies, CAS number 77-86-1). The $\mathrm{pH}$ of the buffer was adjusted to 7.4 with 37 $\%$ hydrochloric acid (Sigma Aldrich, CAS number: 7647-01-0). The solution then was centrifuged at $250 \mathrm{rpm}$ for $1 \mathrm{~h}$ to induce aggregation, at which time the sample still appeared transparent to visual inspection.

\section{Bovine serum albumin}

Solutions of bovine serum albumin (BSA) (Mw: 66,500 Da, Sigma Aldrich, CAS number: $9048-46-8$ ) were aggregated by complexation with poly(allylamine hydrochloride) (PAH) (Mw: 17,500 g/mol, CAS number: 71550-12-4, average degree of polymerization: 1207$)^{36,37}$. BSA and PAH were dissolved in $10 \mathrm{mM}$ Tris- $\mathrm{HCl}$ buffer (pH 7.4) (Life Technologies, CAS number: 77-86-1) to achieve concentrations of 1.22 $\mathrm{mg} / \mathrm{mL}$ and $0.03 \mathrm{mg} / \mathrm{mL}$, respectively. The reagents were mixed by vortexing to ensure dissolution, and aggregates formed after the sample was allowed to equilibrate for one hour.

Additional samples were prepared under comparable conditions with the addition of $0.1 \mathrm{M} \mathrm{NaCl}$ (Sigma Aldrich, CAS number 7647-14-5) to facilitate complexation and thus to promote aggregation.

Control samples were prepared without the addition of salt or $\mathrm{PAH}$, and were measured immediately after preparation.

\section{Preparation of a Stoichiometric Mixture of Colloidal Spheres}

The standard sample of colloidal spheres is constituted as a mixture of four populations of monodisperse colloidal spheres in which each population has a distinct mean size and composition. The monodisperse spheres were purchased from Bangs 
Laboratories as aqueous dispersions at $10 \%$ solids. Stock suspensions were diluted one-thousand-fold with deionized water and then were combined in equal volumes to create a heterogeneous mixture. The four populations in this mixture are polystyrene spheres of diameter $0.71 \pm 0.09 \mathrm{~m}\left(a_{p}=0.36 \pm 0.05 \mathrm{~m}\right)$ (Catalog Code PS03N, Lot Number 9402) and $1.58 \pm 0.14 \mathrm{~m}\left(a_{p}=0.79 \pm 0.07 \mathrm{~m}\right)$ (Catalog Code PS04N, Lot Number 9258), and silica spheres of diameter $0.69 \pm 0.07 \mathrm{~m}\left(a_{p}=0.35 \pm 0.04 \mathrm{~m}\right)$ (Catalog Code SS03N, Lot Number 8933) and $1.54 \pm 0.16 \mathrm{~m}\left(a_{p}=0.77 \pm 0.08 \mathrm{~m}\right)$ (Catalog Code SS04N, Lot Number 5305). The quoted range of particle sizes is estimated by the manufacturer using dynamic light scattering for the smaller spheres, and by the Coulter principle for the larger spheres.

Additional samples of colloidal polystyrene spheres dispersed in water were used to establish the size range for holographic characterization and were not included in the stoichiometric mixture. These samples have nominal radii of of $0.20 \pm$ $0.05 \mathrm{~m}$ (Catalog Code PS02N, Lot Number 6379), $0.78 \pm 0.09 \mathrm{~m}$ (Catalog Code PS04N, Lot Number 9258) and $10.6 \pm 0.7 \mathrm{~m}$ (Catalog Code PS07N, Lot Number 11218).

\section{Preparation of Silicone Spheres}

Silicone spheres composed of polydimethylsiloxane (PDMS) were synthesized by base catalyzed hydrolysis and copolymerization of difunctional diethoxydimethylsilane (DEDMS) (Sigma-Aldrich, CAS number 78-62-6, 3 vol\%) and trifunctional triethoxymethylsilane (TEMS) (Sigma-Aldrich, CAS number 2031-67-6, 2 vol\%) following a standard protocol ${ }^{38,39}$. A mixture of DEDMS and TEMS with 60:40 stoichiometry is added into deionized water (Millipore MilliQ, 93 vol\%) and (28-30) 
$w t \%$ ammonium hydroxide solution (ACROS Organics 2 vol\%) to obtain a total volume of $10 \mathrm{~mL}$. The sample was shaken vigorously with a vortexer for $4 \mathrm{~min}$ at room temperature to initiate nucleation, and then left to polymerize on a rotating frame at 10 rpm for three hours. Fully grown silicone spheres were then mixed with suspensions of protein aggregates at a volume fraction of $10^{-4}$ to obtain an effective concentration of spheres of $4 \times 10^{6} / \mathrm{mL}$.

The polydispersity in radius of these particles can be varied by changing the duration of the mixing process ${ }^{38-40}$. For the present study, we synthesized particles with mean radii around $1.5 \mathrm{~m}$ in one batch with $12 \%$ polydispersity and another with $32 \%$ polydispersity. These different size distributions are intended to have distinctive signatures for holographic characterization.

Lightly polymerized silicone spheres share most properties with unpolymerized silicone oil droplets. Their mean refractive index, $1.388 \pm 0.002$, only slightly exceeds that of DEDMS, 1.381, and TEMS, 1.383, as determined with an Abbe refractometer (Edmund Optics) and by holographic characterization ${ }^{11,26,41}$.

\section{RESULTS}

\section{Verification of Precision, Accuracy and Range}

Figure 2(a) demonstrates the unique and distinguishing ability of holographic characterization to correctly identify distinct populations of colloidal particles in heterogeneous suspensions. These data were obtained through holographic characterization of a model colloidal dispersion consisting of a stoichiometric mixture of four distinct types of monodisperse colloidal spheres. Each of the four clearly resolved peaks in Figure 2(a) represents the properties of one of those populations. 
In each case, the holographically measured distribution of properties is consistent with the manufacturer's specification, which is plotted as a superimposed cross. This agreement, together with complementary tests in previous publications ${ }^{11,15,23,25,26}$, establishes the precision and accuracy of particle-resolved holographic characterization. Specifically, these results demonstrate that this implementation of holographic characterization can yield the radius of an individual micrometer-scale sphere with a precision of $10 \mathrm{~nm}$ and an accuracy of $50 \mathrm{~nm}^{15}$, and the refractive index to within 5 parts per thousand ${ }^{26}$. Previous reports ${ }^{23,24}$ demonstrate comparably good results for porous spheres and otherwise irregular particles.

Figure 2(b) offers an experimental demonstration of the range of particle sizes over which holographic characterization yields useful results. The three holograms presented here were recorded for three different polystyrene spheres dispersed in water, one with a radius of $a_{p}=0.237 \mathrm{~m}$, at the small end of the technique's effective range, one with a radius of $a_{p}=0.800 \mathrm{~m}$, and the third with a radius of $a_{p}=10.47 \mathrm{~m}$. These measured holograms are presented alongside corresponding pixel-by-pixel fits to the predictions of the theory of light scattering, which are parameterized by each particle's three-dimensional position, radius and refractive index. The quality of a fit can be assessed by plotting the radial profile of the normalized image intensity, $b(r)$. This is obtained by averaging the two-dimensional intensity pattern over angles around the center of the scattering pattern. Curves obtained from the measured data are plotted in Figure 2(b) within shaded regions that represent measurement uncertainties. Curves obtained from the fits are overlaid on the experimental data for comparison. The fits track the experimental data extremely well over the entire range 
of particle sizes.

\section{Holographic Characterization of Subvisible Protein Aggregates}

\section{Subvisible Insulin Aggregates}

Although the bovine insulin sample appeared clear under visual inspection, the data in Figure 1 reveal a concentration of $(3.9 \pm 0.1) \times 10^{7}$ subvisible bovine insulin aggregates per milliliter, which corresponds to a volume fraction of roughly $10^{-3}$. Uncertainty in this value results from feature identification errors for the largest particles and uncertainty in the flow speed. Aggregates with radii smaller than $200 \mathrm{~nm}$ are not detected by the holographic characterization system and therefore were not counted in these totals. The distribution of particle characteristics is peaked at a radius of $1.6 \mathrm{~m}$, and is both broad and multimodal. No aggregates were recorded with radii exceeding $4.2 \mathrm{~m}$, up to our detection limit of $10 \mathrm{~m}$, which suggests that such largescale aggregates are present at concentrations below $10^{4} / \mathrm{mL}$.

The aggregates' refractive indexes vary over a wide range from just above that of the buffer, $n_{m}=1.335$, to slightly more than 1.42 . This range is significantly smaller than the value around 1.54 that would be expected for fully dense protein crystals ${ }^{42}$. The observed upper limit is consistent, however, with recent index-matching measurements of the refractive index of protein aggregates ${ }^{43}$. These latter measurements were performed by perfusing protein aggregates with index-matching fluid, and therefore yield an estimate for the refractive index of the protein itself. Holographic characterization, by contrast, analyzes an effective scatterer comprised of both the higher-index protein and also the lower-index buffer that fills out the sphere. We previously have shown that such an effective sphere has an effective refractive 
index intermediate between that of the two media ${ }^{23}$ in a ratio that depends on the actual particle's porosity. More porous or open structures have smaller effective refractive indexes. The influence of porosity on the effective refractive index, furthermore, is found to be proportionally larger for particles with larger radii ${ }^{23}$.

The effective sphere model accounts for general trends in the holographic characterization data under the assumption that the protein aggregates have open irregular structures. This proposal is consistent with previous ex situ studies that have demonstrated that bovine insulin forms filamentary aggregates ${ }^{44-46}$.

The particular ability of holographic characterization to record both the size and the refractive index of individual colloidal particles therefore offers insights into protein aggregates' morphology in situ and without dilution and without any other special preparation. This capability also enables holographic characterization to distinguish protein aggregates from common contaminants such as silicone oil droplets and rubber particles ${ }^{40}$, which pose problems for other analytical techniques ${ }^{47}$.

\section{Subvisible BSA-PAH Complexes}

Figure 3 presents holographic characterization results for two samples of bovine serum albumin complexed with PAH under differing salt concentrations. The data in Figure 3(a) were obtained for the sample prepared without additional salt. As for the insulin sample, holographic characterization of the BSA sample reveals $9.8 \pm 0.5 \times$ $10^{6}$ aggregates $/ \mathrm{mL}$ in the range of radii running from $300 \mathrm{~nm}$ to $2.5 \mathrm{~m}$, and a peak radius of $0.5 \mathrm{~m}$. Although holographic characterization is capable of detecting aggregates with radii up to $10 \mathrm{~m}$, no aggregates were observed with radii exceeding $2.5 \mathrm{~m}$. This suggests that any larger aggregates are present at concentrations below 
$10^{4} / \mathrm{mL}$. The plot range is selected accordingly.

The trace in Figure $3(\mathrm{c})$ is a projection of the joint distribution, $\left(a_{p}, n_{p}\right)$, into the distribution of aggregate sizes, $\left(a_{p}\right)$, obtained by integrating over $n_{p}$. This projection more closely resembles results provided by other characterization techniques, such as micro-flow imaging and dynamic light scattering, that report size data, but no other information.

As for the $\mathrm{BI}$ samples, the anticorrelation between $a_{p}$ and $n_{p}$ evident in Figure 3 suggests that BSA-PAH complexes have an open structure. This is consistent with previous ex situ studies ${ }^{37,48}$ that demonstrate that BSA aggregates into weakly branched structures. Adding salt is known to enhance complexation in the BSA-PAH system $^{36}$. Comparing Figure 3(b) with Figure 3(a) suggests that adding salt increases the mean aggregate size by nearly a factor of two, and also substantially broadens the distribution of aggregate sizes. These trends also can be seen in corresponding projections in Figure 3(b) and Figure 3(d). What the projected size distributions fail to capture is the striking change in the joint distribution of aggregate radii and refractive indexes from Figure 3(a) to Figure 3(b). This shift suggests that the larger aggregates grown in the presence of added salt are substantially more porous ${ }^{23}$. This insight into the aggregates' morphology would not be offered by the size distributions alone.

The number of aggregates detected in Figure $3(b)$ does not differ significantly from the number observed in Figure 3(a). Adding salt therefore appears to increase the size of micrometer-scale subvisible aggregates without appreciably increasing their concentration.

As a control, we performed a holographic characterization measurement on a 
BSA solution with no added PAH or salt, and no deliberate agitation. A 10 min data set reveals a total of 98 features, corresponding to a concentration of $9 \pm 3 \times 10^{5}$ aggregates $/ \mathrm{mL}$ in the accessible size range. This is an order of magnitude fewer aggregates than is observed in the presence of the complexing agent.

\section{Comparison with Established Techniques}

\section{Micro-Flow Imaging}

Figure 4 presents direct comparisons between holographic characterization and micro-flow imaging (MFI) for a representative sample of six BSA-PAH complexes whose morphologies range from nearly spherical compact clusters to extended spindly structures. Each aggregate's hologram is compared with a nonlinear leastsquares fit to the predictions of Lorenz-Mie theory. The reduced ${ }^{2}$ statistic for these fits is used to arrange the results from best fits at the top to worst fits at the bottom. Each of the measured holograms also is used to reconstruct a volumetric image of the individual aggregate through Rayleigh-Sommerfeld deconvolution microscopy ${ }^{32}$. The size of the reconstructed cluster then can be compared with the effective radius obtained from holographic characterization.

Even the two most compact clusters in Figure 4 appear to be substantially aspherical. Their holograms, nevertheless, are very well reproduced by the nonlinear fits. The ${ }^{2}$ metrics for these fits are close to unity, suggesting that the model adequately describes the light-scattering process and that the single-pixel noise is well estimated. Values for the aggregate radius are consistent with the size estimated from RayleighSommerfeld reconstruction. Circles with the holographically-determined radii are superimposed on the numerically refocussed bright-field images in Figure 4(d) for 
comparison. This success is consistent with previous comparisons of Lorenz-Mie and Rayleigh-Sommerfeld analyses for colloidal spheres ${ }^{14}$ and colloidal rods ${ }^{33}$.

Errors increase as aggregates become increasingly highly structured and asymmetric. Even so, estimates for the characteristic size are in reasonable agreement with the apparent size of the bright-field reconstructions even for the worst case. This robustness arises because the effective size of the scatterer strongly influences the size and contrast of the central scattering peak and the immediately surrounding intensity minimum. Faithful fits in this region of the interference pattern therefore yield reasonable values for the scatterer's size. The overall contrast of the pattern as a whole encodes the scatterer's refractive index, and thus is very low for such openstructured clusters.

These representative examples are consistent with earlier demonstrations ${ }^{23,24}$ that holographic characterization yields useful characterization data for imperfect spheres and aspherical particles. Particularly for larger aggregates, the estimated value for the refractive index describes an effective sphere. The estimated radius, however, is a reasonably robust metric for the aggregate's size.

Independent of the ability of holographic characterization to provide insight into morphology, these results demonstrate that holographic microscopy usefully detects and counts subvisible protein aggregates in solution. These detections by themselves provide information that is useful for characterizing the state of aggregation of the protein solution in situ without requiring extensive sample preparation. Holographic microscopy's large effective depth of field then serves to increase the analysis rate relative to particle imaging analysis ${ }^{49}$. 
Like holographic characterization, MFI yields particle-resolved radius measurements that can be used to calculate the concentration of particles in specified size bins. These results may be compared directly with projected size distributions produced by holographic characterization. The data in Figure 5 show such a comparison for the BSA-PAH complexes with and without added salt featured in Figure 3. Results are presented as the number, $N\left(a_{p}\right)$, of aggregates per milliliter in a size range of $\pm 100 \mathrm{~nm}$ around the center of each bin in $a_{p}$. The holographic characterization data from Figure $3(b)$ are rescaled in this plot for comparison. Independent studies demonstrate that MFI analysis yields reliable size estimates for aggregates with radii larger than $1 \mathrm{~m}^{43,50}$. Diffraction causes substantial measurement errors for smaller particles. We therefore collect MFI results for smaller particles into $700 \mathrm{~nm}$-wide bins in Figure 5(a) and Figure 5(b), with corresponding normalization. The lower end of this bin's range corresponds with the smallest radii reported by holographic characterization. Agreement between holographic characterization and MFI is reasonably good over the entire range of particle sizes plotted. Both techniques yield consistent values for the overall concentration of $10^{7}$ aggregates $/ \mathrm{mL}$. MFI systematically reports larger numbers of aggregates on the large end of the size range and fewer on the small end. This difference can be attributed to the most elongated and irregular aggregates, such as the last example in Figure 4, whose size is underestimated by holographic characterization. This effect of morphology on holographic size characterization has been discussed previously ${ }^{24}$. Even in these cases, holographic characterization correctly detects the particles' presence and identifies them as micrometer-scale objects. Both $\mathrm{MFI}$ and holographic 
characterization yield consistent results for the total number density of aggregates.

For particles on the smaller end of the size range, MFI provides particle counts, but no useful characterization data. Holographic characterization, by contrast offers reliable size estimates in this regime. Over the entire range of sizes considered, holographic characterization also provides estimates for particles' refractive indexes.

\section{Dynamic Light Scattering}

To verify the presence of subvisible protein aggregates in our samples, we also performed dynamic light scattering measurements. Whereas holographic characterization and MFI yield particle-resolved measurements, DLS is a bulk characterization technique. Values reported by DLS reflect the percentage, $P\left(a_{h}\right)$, of scattered light that may be attributed to objects of a given hydrodynamic radius, $a_{h}$. The resulting size distribution therefore is weighted by the objects' light-scattering characteristics. Scattering intensities can be translated at least approximately into particle concentrations if the particles are smaller than the wavelength of light and if they all have the same refractive index ${ }^{51}$. Direct comparisons are not possible when particles' refractive indexes vary with size, as is the case for protein aggregates. In such cases, DLS is useful for confirming the presence of scatterers within a range of sizes. Figure 6 presents DLS data for the same samples of BSA-PAH complexes presented in

Figure 3. For both samples, DLS reveals the presence of an abundance of scatterers with radii smaller than $100 \mathrm{~nm}$. The detection threshold of DLS for scatterers of this size is roughly $10^{8}$ aggregates $/ \mathrm{mL}$, as determined by independent studies ${ }^{7}$. We conclude that both samples have at least this concentration of submicrometer- 
diameter aggregates. Such objects are smaller than the detection limit for our implementation of holographic video microscopy, and so were not resolved in Figure 3.

The distribution shifts to larger sizes in the sample with added salt, consistent with the results of holographic characterization. Both samples show a very small signal, indicated by an arrow in Figure 6, for subvisible objects whose hydrodynamic radius is $2.8 \mathrm{~m}$. This confirms the presence of such scatterers in our sample at a concentration just barely above the detection threshold of DLS for objects of that size.

The sample with added salt also has a clear peak around $a_{h}=400 \pm 20 \mathrm{~nm}$ that is in the detection range of holographic characterization. The corresponding peak in Figure $3(b)$ appears at a substantially larger radius, $a_{p}=770 \pm 20 \mathrm{~nm}$. One likely source of this discrepancy is that holographic characterization reports the radius of an effective bounding sphere, whereas DLS reports the hydrodynamic radius, which can be substantially smaller for open structures. Another contributing factor is that larger aggregates have lower effective refractive indexes and thus scatter light proportionately less strongly than smaller aggregates 23,52 . This effect also shifts the apparent size distribution downward in DLS measurements. It does not, however, affect holographic characterization, which reports both the size and refractive index of each object independently.

\section{Holographic Differentiation of Silicone Spheres from Protein Aggregates}

DLS cannot distinguish protein aggregates from other populations of particles in suspension. MFI can differentiate some such contaminants by morphology: silicone droplets, for example, tend to be spherical, whereas protein aggregates tend to have 
irregular shapes. Morphological differentiation works best for particles that are substantially larger than the wavelength of light, whose structural features are not obscured by diffraction. Through the information it provides on individual particles' refractive indexes, holographic characterization offers an additional avenue for distinguishing micrometer-scale objects by composition. We demonstrate this capability by performing holographic characterization measurements on BSA samples that are deliberately adulterated with silicone spheres.

\section{Holographic Characterization of Silicone Spheres}

Figure 7 shows holographic characterization data for silicone spheres dispersed in deionized water. The sample in Figure $7(a)$ is comparatively monodisperse with a sample-averaged radius of $0.75 \pm 0.09 \mathrm{~m}$. The particles in Figure $7(\mathrm{~b})$ are drawn from a broader distribution of sizes, with a mean radius of $0.87 \pm 0.28 \mathrm{~m}$. Both samples of spheres have refractive indexes consistent with previously reported values for PDMS with $40 \%$ crosslinking, $n_{p}=1.388 \pm 0.005^{40}$. This range is indicated with a shaded region in Figure 7.

Unlike the protein aggregates, these particles' refractive indexes are uncorrelated with their sizes. This is most easily seen in the polydisperse sample in Figure $7(b)$, and is consistent with the droplets having uniform density and no porosity ${ }^{40}$. We expect to see the same distribution of single-particle properties when these silicone spheres are co-dispersed with protein aggregates.

\section{Differential Detection of Silicone Spheres}

The data in Figure 8(a) were obtained from a sample of BSA prepared under the same conditions as Figure 3(a), but with the addition of monodisperse silicone 
spheres at a concentration of $4 \times 10^{6}$ particles $/ \mathrm{mL}$. The resulting distribution of particle properties is clearly bimodal with one population resembling that obtained from protein aggregates alone, and the other having a refractive index consistent with that of the silicone spheres, $n_{p}=1.388 \pm 0.005^{41}$. The sample in Figure $8(\mathrm{~b})$ similarly were prepared under conditions comparable to those from Figure $3(b)$ with the addition of polydisperse silicone spheres from the sample in Figure $7(b)$. Consistency between features associated with protein aggregates in Figure 3 and Figure 8 demonstrate that both the sample preparation protocol and also the holographic characterization technique yield reproducible results from sample to sample, and that holographic characterization of protein aggregates is not influenced by the presence of extraneous impurity particles.

Interestingly, both distributions feature a small peak around $a_{p}=2.8 \mathrm{~m}$ that corresponds to the peak in the DLS data from Figure 6 . This feature is not present in Figure 3. It is likely that this very small population of larger aggregates was present in those samples, but at a concentration just below the threshold for detection in a 10 min measurement.

The distributions of features associated with silicone droplets in Figure 8 also agree well with the holographic characterization data on the droplets alone from Figure 7 . These results demonstrate that the refractive-index data from holographic characterization can be useful for distinguishing protein aggregates from silicone oil droplets. Such differentiation would not be possible on the basis of the size distribution alone, as can be seen from the projected data in Figure 8.

Holographic characterization cannot differentiate silicone droplets from protein 
clusters whose refractive index is the same as silicone's. Such ambiguity arises for the smallest particles analyzed in Figure 8. Spherical silicone droplets sometimes can be distinguished from irregularly shaped protein aggregates under these conditions using morphological data obtained through deconvolution analysis of the same holograms. The distinction in these cases still would be less clear than can be obtained with Resonant Mass Measurement (RMM), which differentiates silicone from protein by the sign of their relative buoyancies ${ }^{50}$. In cases where specific particles cannot be differentiated unambiguously, the presence of silicone droplets still can be inferred from holographic characterization data because such particles create a cluster in the $\left(a_{p}, n_{p}\right)$ plane whose refractive index is independent of size. The relative abundances of the two populations then can be inferred, for example, by statistical clustering methods.

\section{DISCUSSION}

\section{Comparison with Established Techniques}

Among available particle characterization techniques, holographic characterization is unique in its ability to measure both the size and the refractive index of individual particles. In other respects, it complements established techniques.

As an imaging technique, holographic characterization is related to micro-flow imaging (MFI) ${ }^{9}$ and nanoparticle tracking analysis (NTA) ${ }^{49}$. Both MFI and holographic characterization can characterize a single particle with a single snapshot, and so are inherently faster than NTA, which relies on time-series analysis of a particle's Brownian motion to estimate its hydrodynamic radius. This analysis requires information on the solvent's viscosity, which is not required for MFI or holographic 
characterization. NTA is best suited for sizing sub-micrometer particles, which diffuse quickly ${ }^{49}$. MFI is most effective for the largest subvisible particles ${ }^{9}$. Holographic characterization bridges the gap.

Holographic characterization also is related to light-scattering techniques such as dynamic light scattering (DLS) $)^{7,8,49,51}$. Like NTA, DLS is sensitive to smaller particles than holographic characterization. As a sample-averaged measurement, however, DLS does not inherently account for heterogeneity in sample composition. DLS cannot differentiate silicone droplets from protein aggregates, for example. Estimating relative abundances of particles with different sizes, furthermore, requires information such as the particles' refractive indexes and the solvent's viscosity to be provided as inputs. Particle-resolved characterization techniques, including NTA, MFI and holographic characterization, therefore provide more reliable information on size distributions, particularly in heterogeneous samples. Single-particle methods also are sensitive to lower concentrations of particles than DLS.

By measuring the degree to which individual particles block a beam of light, light obscuration (LO) combines some benefits of light scattering and particle imaging ${ }^{43,47}$. Typically used for particles larger than $2 \mathrm{~m}$, LO requires particles to be well-enough separated in the flow to be detected individually. Samples with concentrations higher than $2 \times 10^{4}$ particles $/ \mathrm{mL}$ may have to be diluted into this operating range $\mathrm{e}^{47}$. Light obscuration cannot distinguish different types of particles in heterogeneous samples, and so cannot differentiate protein aggregates from silicone oil.

As an optical probe of protein aggregates' properties, holographic characterization is orthogonal to such non-optical techniques as the Coulter principle 
and Resonant Mass Measurement (RMM), both of which are particle-resolved measurement techniques. Coulter counters yield measurements of particles' volumes over a size range determined by the choice of aperture through which the particles flow. Clogging can pose problems ${ }^{47}$. Like LO, the Coulter principle requires particles to pass through the measurement volume one at a time, which sets an upper limit on particle concentration of $1 \times 10^{5}$ particles $/ \mathrm{mL}$. In addition, Coulter counters impose requirements on the solution's conductivity. Achieving operating conditions may require changes to proteins' chemical environment that influences their aggregation behavior 47 .

RMM measures the buoyant mass of individual particles by their influence on the resonant frequency of the microfluidic channel through which they flow $7,43,50$. This approach naturally differentiates objects such as silicone droplets that are less dense than water from objects such as protein aggregates that are more dense. It cannot, however, differentiate materials with the same sign of the buoyant mass, such as protein and rubber. Because it requires particles to pass through a narrow channel, RMM analyzes smaller sample volumes per unit time than holographic characterization, and so cannot easily probe sample concentrations below $1 \times 10^{6}$ particles $/ \mathrm{mL}$. As a measurement of buoyant mass, moreover, RMM does not directly report particle size.

Comparisons among these techniques are summarized in Table I.

\section{Summary of Key Points}

The measurements presented here demonstrate that holographic video microscopy together with Lorenz-Mie analysis can detect, count and characterize 
subvisible protein aggregates. Data acquisition is rapid, typically taking no more than 15 min, and requires no special sample preparation. Our implementation is effective for colloidal particles ranging in radius from $300 \mathrm{~nm}$ to $10 \mathrm{~m}$ and at concentrations from $10^{4}$ particles $/ \mathrm{mL}$ to $10^{8}$ particles $/ \mathrm{mL}$.

Large irregularly shaped protein aggregates can be mistaken for smaller aggregates by our present implementation of holographic characterization. Such cases are readily detected through the quality of fit and can be remedied by micro-flow imaging analysis of the same holograms. This additional analysis step also provides information on the morphology of individual protein aggregates.

Instrumentation for holographic characterization is compact and closely resembles the optical train for a conventional light microscope. Calibration requires values for the laser wavelength, the microscope's magnification and the medium's refractive index. The measurement process similarly is straightforward, requiring less than one hundred microliters of sample. Data recording and analysis is entirely automated.

We anticipate, therefore, that holographic characterization of protein aggregates will be useful for assessing the stability of biopharmaceutical formulations throughout the product lifecycle. Access to time-resolved characterization data for micrometerscale subvisible protein aggregates should prove useful for assessing the efficacy of stabilizing agents during product development. Automated continuous operation lends itself to process control for manufacturing. Variants of holographic characterization might even lend themselves to assessing the quality of biopharmaceutical products in situ. 


\section{ACKNOWLEDGMENTS}

This work was supported by the MRSEC program of the National Science Foundation under Award Number DMR-1420073. The holographic characterization system was developed with support of the MRI program of the NSF under Award Number DMR-0922680. The authors are grateful to Jaroslaw Blusewicz for acquiring the data for Figure 2.

\section{REFERENCES}

${ }^{1}$ Food and Drug Administration. Guidance for Industry: Immunogenicity Assessment for Therapeutic Protein Products. U.S. Department of Health and Human Services; 2014.

2 Schellekens $\mathrm{H}$ 2002. Bioequivalence and the immunogenicity of biopharmaceuticals. Nat Rev Drug Discov. 1:457-462.

3 Wang W 2005. Protein aggregation and its inhibition in biopharmaceutics. Int $\mathrm{J}$ Pharma 289:1-30.

${ }^{4}$ Carpenter JF, Randolph TW, Jiskoot W, Commelin DJA, Middaugh CR, Winter G, Fan YX, Kirshner S, Verthelyi D, Kozlowski S, Clouse KA, Swann PG, Rosenberg A, Cherney B 2009. Overlooking subvisible particles in therapeutic protein products: Gaps that may compromise product quality. J Pharm Sci 4:1201-1205.

5 Singh SK, Afonina N, Awwad M, Bechtold-Peters K, Blue JT, Chou D, Cromwell M, Krause HJ, Mahler HC, Meyer, BK, Narhi L, Nesta DP, Spitznagel, T 2010. An industry perspective on the monitoring of subvisible particles as a quality attribute for protein therapeutics. J Pharm Sci 99:3302-3321.

6 den Englesman J, Gandel P, Smulders R, Koll H, Smith B, Bassarab S, Seidl A, 
Hainzl O, Jiskoot, W 2011. Strategies for the assessment of protein aggregates in pharmaceutical biotech product development. Pharm Res 28:920-933.

7 Panchal J, Kotarek J, Marszal E, Topp EM 2014. Analyzing subvisible particles in protein drug products: A comparison of Dynamic Light Scattering (DLS) and Resonant Mass Measurement (RMM). AAPS J 16(3):440-451.

8 Hamrang Z, Hussain M, Tingey K, Tracka M, Casas-Finet J, Uddin S, van der Walle, CF, Pluen, A 2015. Characterization of stress-induced aggregate size distributions and morphological changes of a bi-specific antibody using orthogonal techniques. J Pharm Sci 104(8):2473-2481.

9 Sharma DK, King D, Oma P, Merchant C 2015. Micro-Flow Imaging: Flow microscopy applied to sub-visible particulate analysis in protein formulations. AAPS J 12(3):455-464.

10 Zölls S, Wiggenhorn M, Winter G, Friess W, Jiskoot W 2013. Flow imaging microscopy for protein particle analysis - A comparative evaluation of four different analytical instruments. AAPS J 15(4):1200-1211.

11 Lee SH, Roichman Y, Yi GR, Kim SH, Yang SM, van Blaaderen A, van Oostrum P, Grier DG 2007. Characterizing and tracking single colloidal particles with video holographic microscopy. Opt Express 15:18275-18282.

12 Sheng J, Malkiel E, Katz J 2006. Digital holographic microscope for measuring three-dimensional particle distributions and motions. Appl Opt 45(16):3893-3901.

13 Lee SH, Grier DG 2007. Holographic microscopy of holographically trapped threedimensional structures. Opt Express 15:1505-1512. 
14 Cheong FC, Krishnatreya BJ, Grier DG 2010. Strategies for three-dimensional particle tracking with holographic video microscopy. Opt Express 18:13563-13573.

15 Krishnatreya BJ, Colen-Landy A, Hasebe P, Bell BA, Jones JR, Sunda-Meya A, Grier DG 2014. Measuring Boltzmann's constant through holographic video microscopy of a single sphere. Am J Phys 82:23-31.

16 Bohren CF, Huffman DR 1983. Absorption and Scattering of Light by Small Particles. New York: Wiley Interscience.

17 Mishchenko MI, Travis LD, Lacis AA 2011. Scattering, Absorption and Emission of Light by Small Particles. Cambridge: Cambridge University Press.

18 Gerard G, Gréhan G 2011. Generalized Lorenz-Mie Theories. Berlin: SpringerVerlag.

19 Cheong FC, Sun B, Dreyfus R, Amato-Grill J, Xiao K, Dixon L, Grier DG 2009. Flow visualization and flow cytometry with holographic video microscopy. Opt Express 17:13071-13079.

20 Fung J, Martin KE, Perry RW, Kaz DM, McGorty R, Manoharan VN 2011. Measuring translational, rotational, and vibrational dynamics in colloids with digital holographic microscopy. Opt Express 19:8051-8065.

21 Perry RW, Meng GN, Dimiduk TG, Fung J, Manoharan VN 2012. Real-space studies of the structure and dynamics of self-assembled colloidal clusters. Faraday Discuss 159:211-234.

22 Fung J, Perry RW, Dimiduk TG, Manoharan VN 2012. Imaging multiple colloidal particles by fitting electromagnetic scattering solutions to digital holograms. J Quant 
Spectr Rad Trans 113:2482-2489.

23 Cheong FC, Xiao K, Pine DJ, Grier DG 2011. Holographic characterization of individual colloidal spheres' porosities. Soft Matter 7:6816-6819.

24 Hannel M, Middleton C, Grier DG 2015. Holographic characterization of imperfect colloidal spheres. Appl Phys Lett 107:141905.

25 Moyses H, Krishnatreya BJ, Grier DG 2013. Robustness of holographic video microscopy against defects in illumination. Opt Express 21:5968-5973.

26 Shpaisman H, Krishnatreya BJ, Grier DG 2012. Holographic microrefractometer. Appl Phys Lett 101:091102.

27 Krishnatreya BJ, Grier DG 2014. Fast feature identification for holographic tracking: The orientation alignment transform. Opt Express 22:12773-12778.

28 Yevick A, Hannel M, Grier DG 2014. Machine-learning approach to holographic particle characterization. Opt Express 22:26884-26890.

29 Dixon L, Cheong FC, Grier DG 2011. Holographic particle-streak velocimetry. Opt Express 19:4393-4398.

30 Crocker JC, Grier DG 1996. Methods of digital video microscopy for colloidal studies. J Colloid Interface Sci 179:298-310.

31 Silverman BW 1992. Density Estimation for Statistics and Data Analysis. New York: Chapman \& Hall.

32 Dixon L, Cheong FC, Grier DG 2011. Holographic deconvolution microscopy for high-resolution particle tracking. Opt Express 19:16410-16417.

33 Cheong FC, Grier DG 2010. Rotational and translational diffusion of copper oxide 
nanorods measured with holographic video microscopy. Opt Express 18:6555-6562.

34 Sluzky V, Tamada JA, Klibanov AM, Langer R 1991. Kinetics of insulin aggregation in aqueous solutions upon agitation in the presence of hydrophobic surfaces. Proc Natl Acad Sci USA 88(21):9377-9381.

35 Costantino HR, Langer R, Klibanov AM 1994. Moisture-induced aggregation of lyophilized insulin. Pharm Res 11:21-29.

36 Ball V, Winterhalter M, Schwinte P, Lavalle P, Voegel JC, Schaaf P 2002. Complexation mechanism of bovine serum albumin and poly(allylamine hydrochloride). J Phys Chem B 106:2357-2364.

37 Hagiwara T, Kumagai H, Nakamura K 1996. Fractal analysis of aggregates formed by heating dilute BSA solutions using light scattering methods. Biosci Biotech Biochem 60(11):1757-1763.

38 Obey TM, Vincent B 1994. Novel monodisperse "silicone oil"/water emulsions. J Colloid Interface Sci 163:454-463.

39 Goller MI, Obey TM, Teare DOH, Vincent B, Wegener MR 1997. Inorganic "silicone oil" microgels. Colloids Surfaces A 123-124:183-193.

40 Wang C, Shpaisman H, Hollingsworth AD, Grier DG 2015. Monitoring colloidal growth with holographic microscopy. Soft Matter 11:1062-1066.

41 Wang C, Moyses HW, Grier DG 2015. Stimulus-responsive colloidal sensors with fast holographic readout. Appl Phys Lett 107:051903.

42 Maschke A, Calí N, Appel B, Kiermaier J, Blunk T, Göpferich A 2006. Micronization of insulin by high pressure homogenization. Pharm Res 23(9):2220-2229. 
43 Zölls S, Gregoritza M, Tantipolphan R, Wiggenhorn M, Winter G, Friess W, Hawe A 2013. How subvisible particles become invisible - Relevance of the refractive index for protein particle analysis. J Pharm Sci 102(5):1434-1446.

44 Omichi M, Asano A, Tsukuda S, Takano K, Sugimoto M, Saeki A, Sakamaki D, Onoda A, Hayashi T, Seki S 2014. Fabrication of enzyme-degradable and sizecontrolled protein nanowires using single particle nano-fabrication technique. Nature Commun 5:3717.

45 Yip CM, Brader ML, DeFelippis MR, Ward MD 1998. Atomic Force Microscopy of crystalline insulin: the influence of sequence variation and interfacial structure. Biophys J 74:2199-2209.

46 Yip CM, DeFelippis MR, Frank BH, Brader ML, Ward MD 1998. Structural and morphological characterization of ultralente insulin crystals by Atomic Force Microscopy: evidence of hydrophobically driven assembly. Biophys J 75:1172-1179.

47 Demeule B, Messick S, Shire SJ, Liu J 2010. Characterization of particles in protein solutions: Reaching the limits of current technologies. AAPS J 12(4):708-715.

48 Siposova K, Kubovcikova M, Bednarikova Z, Koneracka M, Savisova V, Antosova A, Kopcansky P, Daxnerova Z, Gazova Z 2012. Depolymerization of insulin amyloid fibrils by albumin-modified magnetic field. Nanotech 23(5):055101.

49 Filipe V, Hawe A, Jiskoot W 2010. Critical evaluation of nanoparticle tracking analysis (NTA) by NanoSight for the measurement of nanoparticles and protein aggregates. Pharm Res 27:796-810.

50 Weinbuch D, Zölls S, Wiggenhorn M, Friess W, Winter G, Jiskoot W, Hawe A 2013. 
Micro-flow imaging and resonant mass measurement (archimedes) - complementary methods to quantitatively differentiate protein particles and silicone oil droplets. $\mathrm{J}$ Pharm Sci 102(7):2152-2165.

51 Berne BJ, Pecora R 1976. Dynamic Light Scattering with Application to Chemistry, Biology and Physics. New York: Courier Dover.

52 Feder J, Jossang T, Rosenqvist E 1984. Scaling behavior and cluster fractal dimension determined by light-scattering from aggregating proteins. Phys Rev Lett 53:1403-1406. 


\section{FIGURE CAPTIONS}

Figure 1. Protein aggregates flowing down a microfluidic channel form holograms as they pass through a laser beam. A typical experimental hologram is reproduced as a grayscale image in the figure. Each hologram is recorded by a video camera and compared with predictions of Lorenz- Mie theory to measure each aggregate's radius, $a_{p}$, and refractive index, $n_{p}$. The scatter plot shows experimental data for 3000 subvisible aggregates of bovine insulin, with each data point representing the properties of a single aggregate, and colors denoting the relative probability density $\left(a_{p}, n_{p}\right)$ for observations in the $\left(a_{p}, n_{p}\right)$ plane.

Figure 2. (a) Scatter plot of radius $a_{p}$ and refractive index $n_{p}$ obtained with holographic characterization of the four-component stoichiometric colloidal mixture described in the Materials section. Results for 20,000 particles are plotted. Superimposed crosses indicate the manufacturer's specification for each of the four populations. These results establish holographic characterization's ability to differentiate particles by composition as well as by size. (b) Measured holograms of colloidal polystyrene spheres in water together with fits, demonstrating the range of particle sizes amenable to holographic characterization. These typical examples were obtained for spheres with radii $a_{p}=$ $0.237 \mathrm{~m}(224$ pixel $\times 224$ pixel region of interest), $0.800 \mathrm{~m}(356$ pixel $\times 356$ pixel $)$ and $10.47 \mathrm{~m}$ (608 pixel $\times 608$ pixel). The fit to each hologram yields values for the particle's radius, $a_{p}$, and refractive index, $n_{p}$. Radial profiles, $b(\underline{r})$, are obtained from these holograms and their fits by averaging the normalized intensity over angles around the center of each feature, and are plotted as a function of distance $r$ from the center of the feature. Experimental data are plotted as blue curves within shaded 
regions representing the measurement's uncertainty at that radius. Fits are superimposed as orange curves and closely track the experimental data.

Figure 3. Influence of added salt on the measured distribution of the radius $a_{p}$ and refractive index $n_{p}$ of BSA-PAH complexes. Each point in the scatter plots represents the properties of a single aggregate and is colored by the relative density of observations, $\left(a_{p}, n_{p}\right)$. (a) BSA complexed with PAH in Tris buffer (1100 aggregates). (b) The same sample with $0.1 \mathrm{M} \mathrm{NaCl}$ (1200 aggregates). (c) and (d) present the projected relative size distributions, $\left(a_{p}\right)$, from (a) and (b), respectively. Shaded regions represent the instrumental and statistical error.

Figure 4. Influence of aggregate morphology on holographic characterization. Holograms of typical aggregates arranged in order of increasing discrepancy between measured and fit holograms. Column (a) shows 160 pixel $\times 160$ pixel regions of interest from the microscope's field of view, centered on features automatically identified as candidate BSA-PAH complexes. Column (b) shows fits to the Lorenz-Mie theory for holograms formed by spheres. Column (c) shows radial profiles of the experimental hologram (black curves) overlaid with the radial profile of the fits (red curves). Shaded regions represent the estimated experimental uncertainty. Column (d) shows Rayleigh-Sommerfeld reconstructions of the aggregates' three-dimensional structures obtained from the experimental holograms. Grayscale images are projections of the reconstructions, which resemble equivalent bright-field images at optimal focus. Superimposed circles indicate fit estimates for the particle size.

Figure 5. Comparison of size distributions measured with Micro-Flow Imaging and holographic characterization. Each bin represents the number of particles per milliliter 
of solution in a range of $\pm 100 \mathrm{~nm}$ about the bin's central radius. (a) Sample without added salt from Figure 3(a). (b) Same with added $\mathrm{NaCl}$ from Figure 3(b).

Figure 6. Characterization of BSA-PAH complexes by dynamic light scattering (DLS). Values represent the percentage, $P\left(a_{h}\right)$, of the scattered light's intensity due to scatterers of a given hydrodynamic radius, $a_{h}$. The arrow indicates a small peak in both distributions around $a_{h}=2.8 \mathrm{~m}$.

Figure 7. Holographic characterization data for silicone spheres dispersed in deionized water. The gray-shaded region denotes range of refractive indexes expected for these particles based on their composition. (a) Monodisperse sample (600 spheres). (b) Polydisperse sample (600 spheres).

Figure 8. Holographic measurement of the relative probability density, $\left(a_{p}, n_{p}\right)$, of particle radius and refractive index for suspensions BSA-PAH complexes spiked with added silicone spheres. (a) Sample prepared under the same conditions as in Figure 3(a) spiked with the monodisperse spheres from Figure 7(a) (2000 features). (b) Sample prepared under the same conditions as in Figure 3(b) spiked with the polydisperse spheres from Figure 7(b) (1600 features). (c) and (d) show the projected relative probability density, $\left(a_{p}\right)$, for particle radius from the data in (a) and (b), respectively. 


\section{TABLE CAPTION}

TABLE I. Comparison of high-throughput characterization techniques for subvisible protein aggregates. The size range refers to the radius of the effective sphere detected by each method. The fourth column indicates whether the technique is capable of measuring aggregate morphology. The term "differentiation" in the fifth column refers to a technique's ability to distinguish particles of similar size but different composition. References describe independent assessments of techniques' capabilities for characterizing protein aggregates. 


\begin{tabular}{|c|c|c|c|c|}
\hline Method & Size $[\mu \mathrm{m}]$ & Number/mL & Morphology & Comments \\
\hline Holographic Characterization & $0.3-10$ & $10^{4}-10^{8}$ & Yes & $\begin{array}{l}\text { Measures both size and refractive } \\
\text { index. } \\
\text { Differentiates by refractive index. } \\
\text { Can differentiate by morphology. }\end{array}$ \\
\hline $\begin{array}{l}\text { Dynamic Imaging Analysis (DIA) } \\
\text { Micro-Flow Imaging (MFI) } 39,47\end{array}$ & $1-400$ & $10^{4}-10^{6}$ & Yes & Differentiates by morphology. \\
\hline $\begin{array}{l}\text { Dynamic Light Scattering } \\
(\text { DLS })^{27,45}\end{array}$ & $0.001-1$ & $10^{8}-10^{12}$ & No & $\begin{array}{l}\text { Sample-averaged measurement. } \\
\text { No differentiation. }\end{array}$ \\
\hline $\begin{array}{l}\text { Nanoparticle Tracking Analysis } \\
(\text { NTA })^{27}\end{array}$ & $0.03-1$ & $10^{7}-10^{9}$ & No & $\begin{array}{l}\text { Measurement time increases with } \\
\text { particle radius. } \\
\text { No differentiation. }\end{array}$ \\
\hline Light Obscuration (LO) ${ }^{39,44}$ & $1-200$ & $10^{3}-10^{5}$ & No & $\begin{array}{l}\text { Typically requires sample dilution. } \\
\text { Sensitive to refractive index } \\
\text { variations. } \\
\text { Requires calibration with size } \\
\text { standards. } \\
\text { No differentiation. }\end{array}$ \\
\hline $\begin{array}{l}\text { Electric Sensing Zone (ESZ) } \\
\text { Coulter Principle } 44\end{array}$ & $0.1-1600$ & $1-10^{5}$ & No & $\begin{array}{l}\text { Requires compatible electrolyte. } \\
\text { Typically requires sample dilution. } \\
\text { Requires calibration with size } \\
\text { standards. } \\
\text { Size range determined by orifice. } \\
\text { No differentiation. }\end{array}$ \\
\hline $\begin{array}{l}\text { Resonant Mass Measurement } \\
\text { (RMM) Archimedes } 39,45,47\end{array}$ & $0.3-4$ & $10^{6}-10^{9}$ & No & $\begin{array}{l}\text { Particle size estimated indirectly } \\
\text { from mass. } \\
\text { Differentiates between positively- } \\
\text { and negatively-buoyant particles }\end{array}$ \\
\hline
\end{tabular}


Figures 1

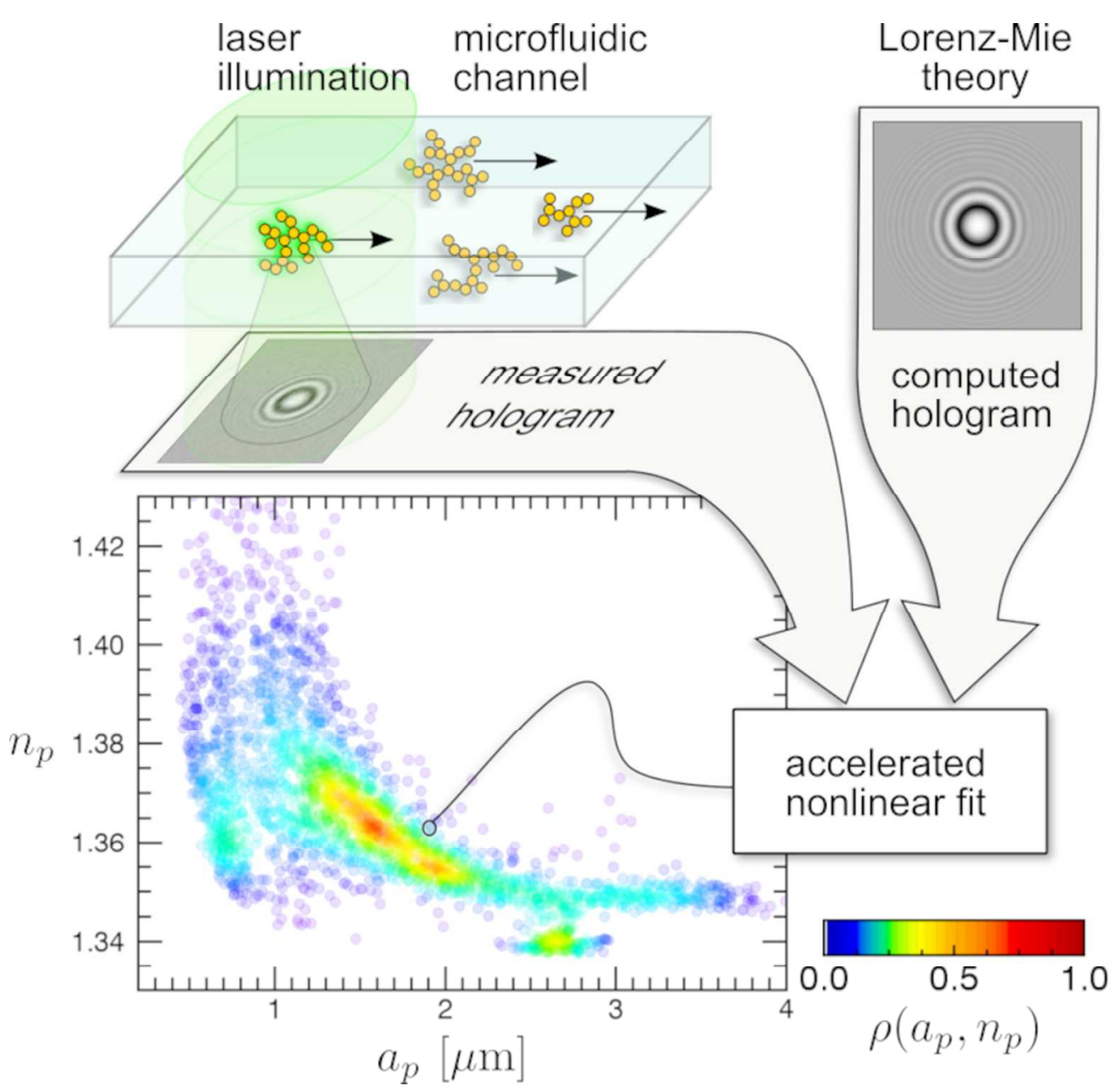

$84 \times 80 \mathrm{~mm}(300 \times 300 \mathrm{DPI})$

John Wiley \& Sons, Inc. 

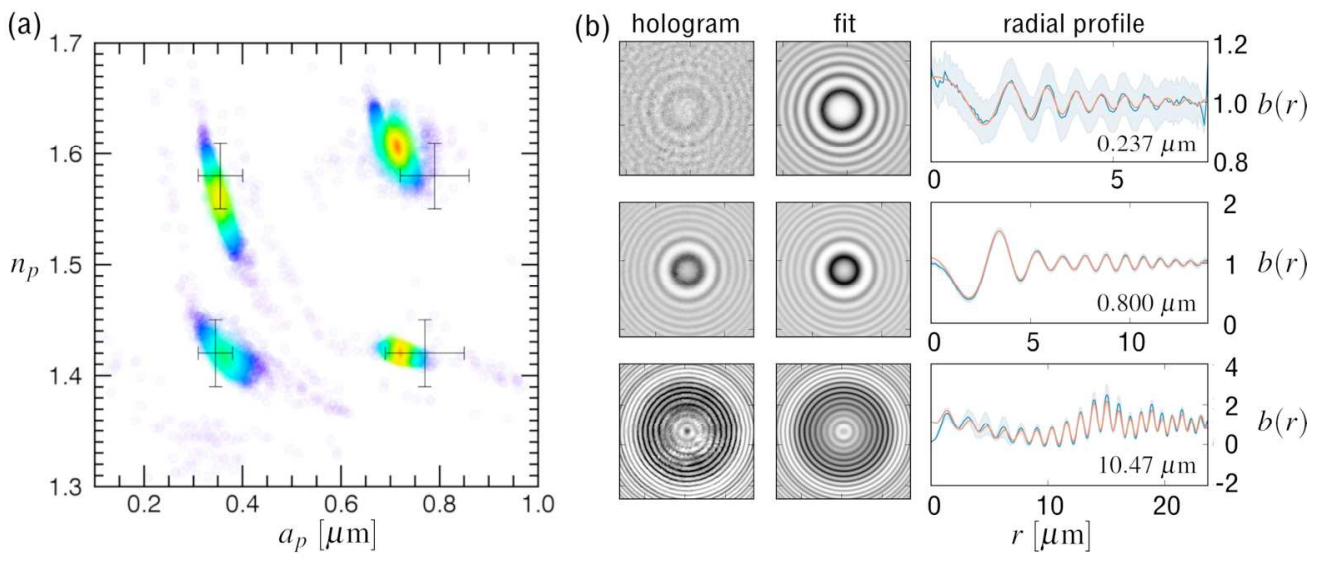

$177 \times 75 \mathrm{~mm}(300 \times 300$ DPI $)$ 


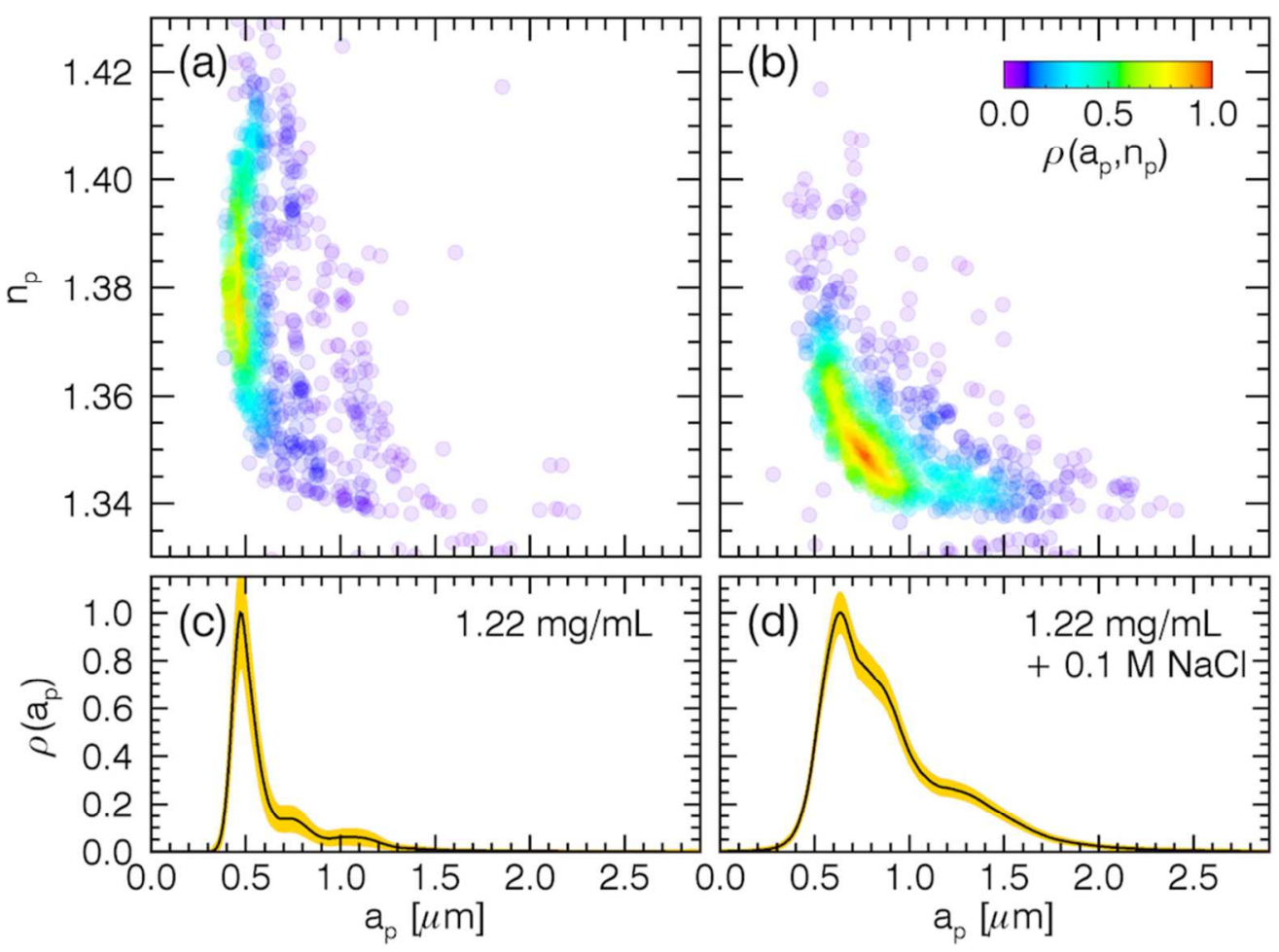

$84 \times 62 \mathrm{~mm}(300 \times 300$ DPI $)$

John Wiley \& Sons, Inc. 
(a) hologram

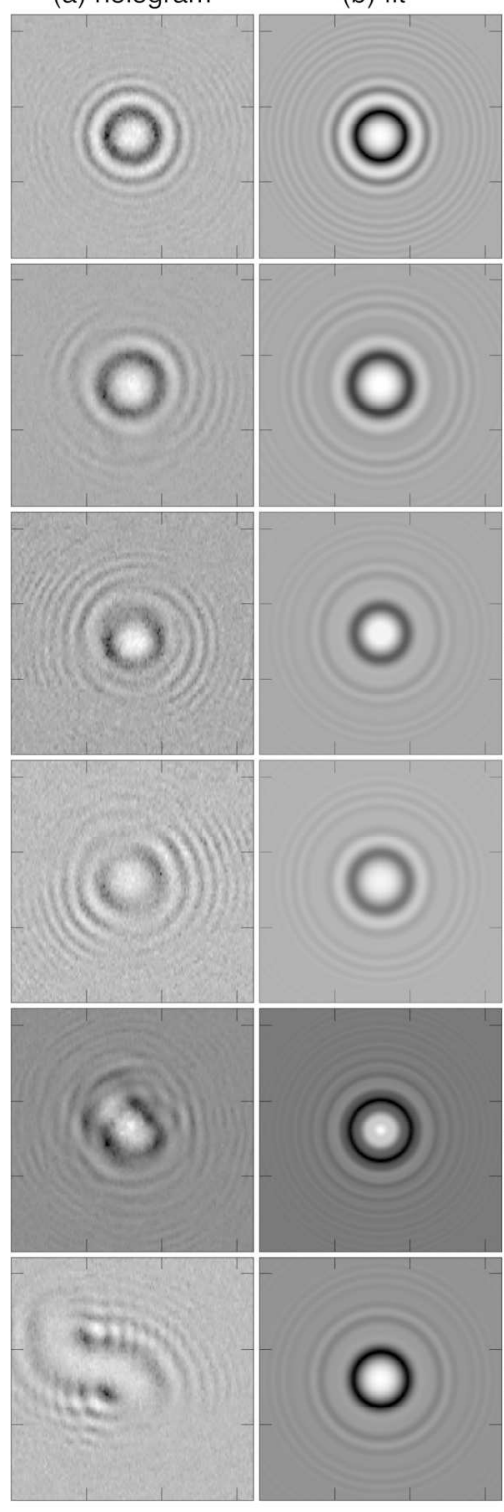

(c) radial profile

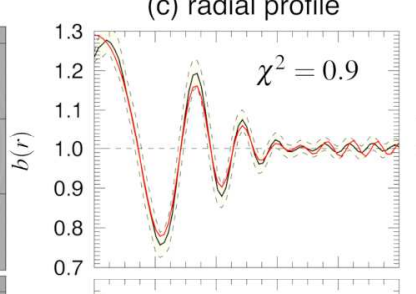

(d) reconstruction

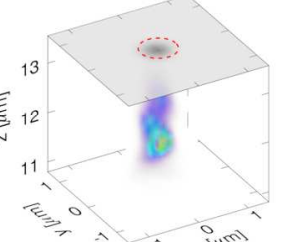

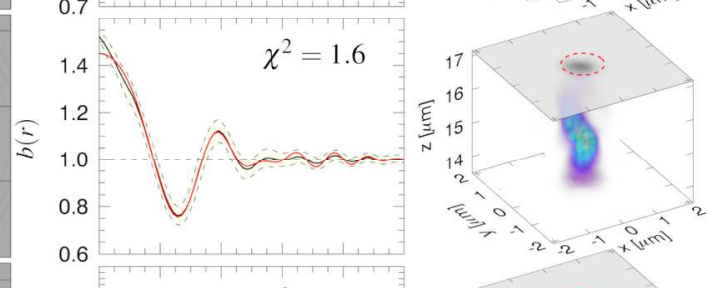
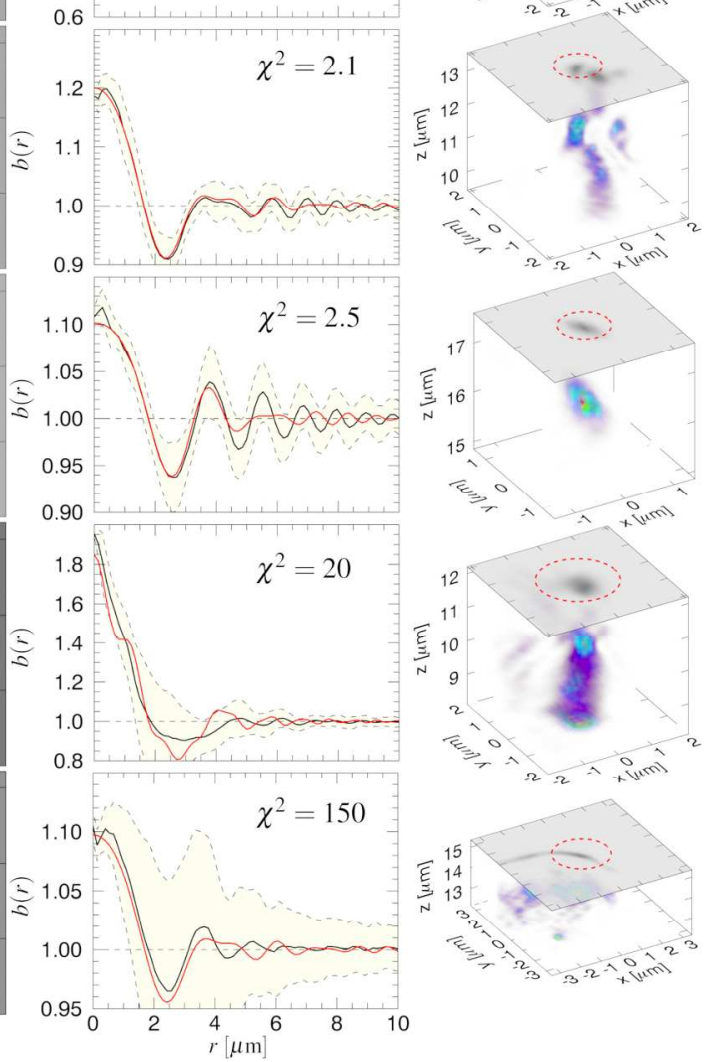

$177 \times 233 \mathrm{~mm}(300 \times 300$ DPI $)$

John Wiley \& Sons, Inc. 
Comparison of size distributions measured with Micro-Flow Imaging and holographic characterization. Each bin represents the number of particles per milliliter of solution in a range of $\pm 100 \mathrm{~nm}$ about the bin's central radius. (a) Sample without added salt from Figure 3(a). (b) Same with added $\mathrm{NaCl}$ from Figure 3(b). $69 \times 84 \mathrm{~mm}(300 \times 300 \mathrm{DPI})$ 


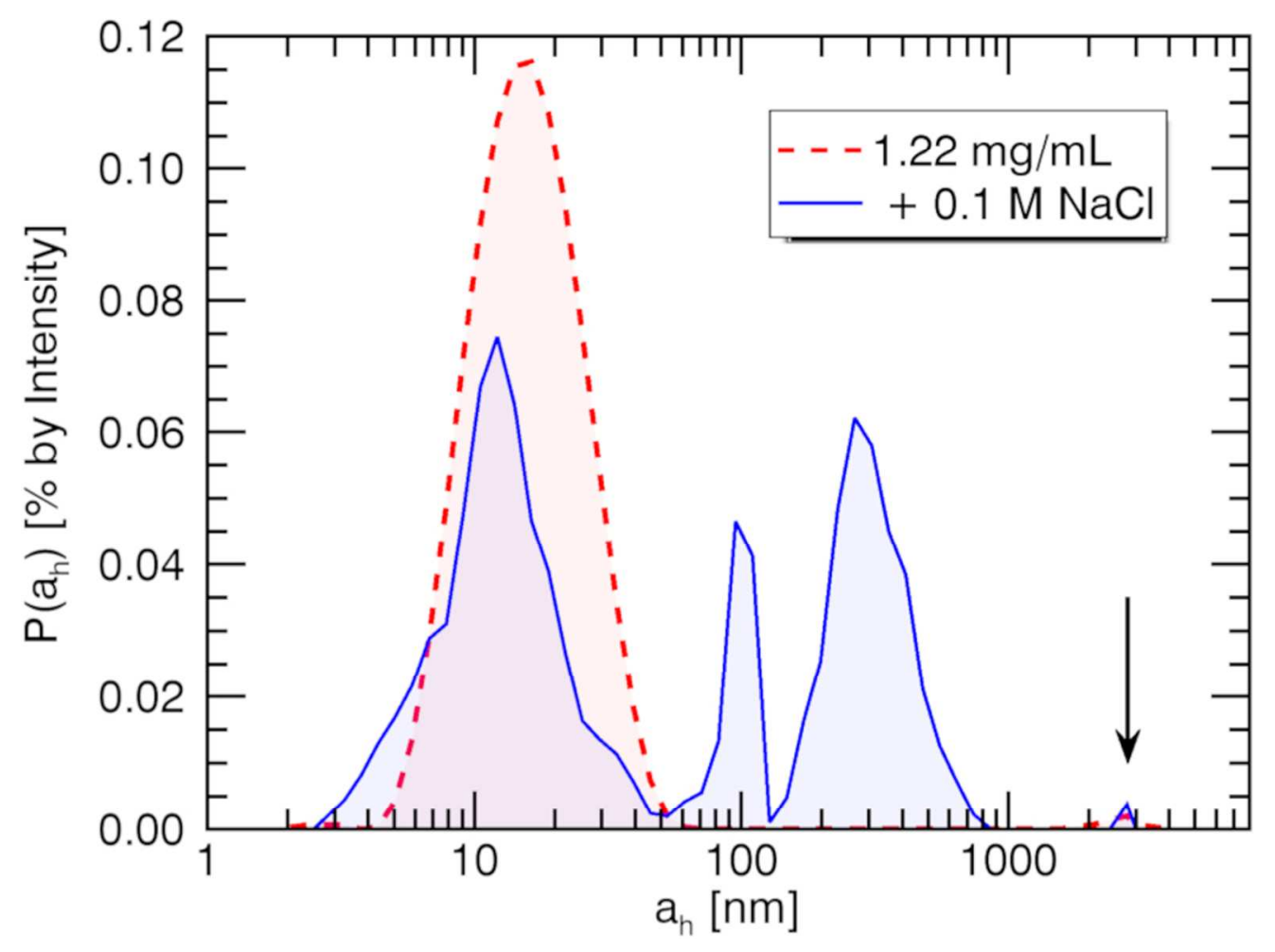

$84 \times 63 \mathrm{~mm}(300 \times 300$ DPI $)$

John Wiley \& Sons, Inc. 


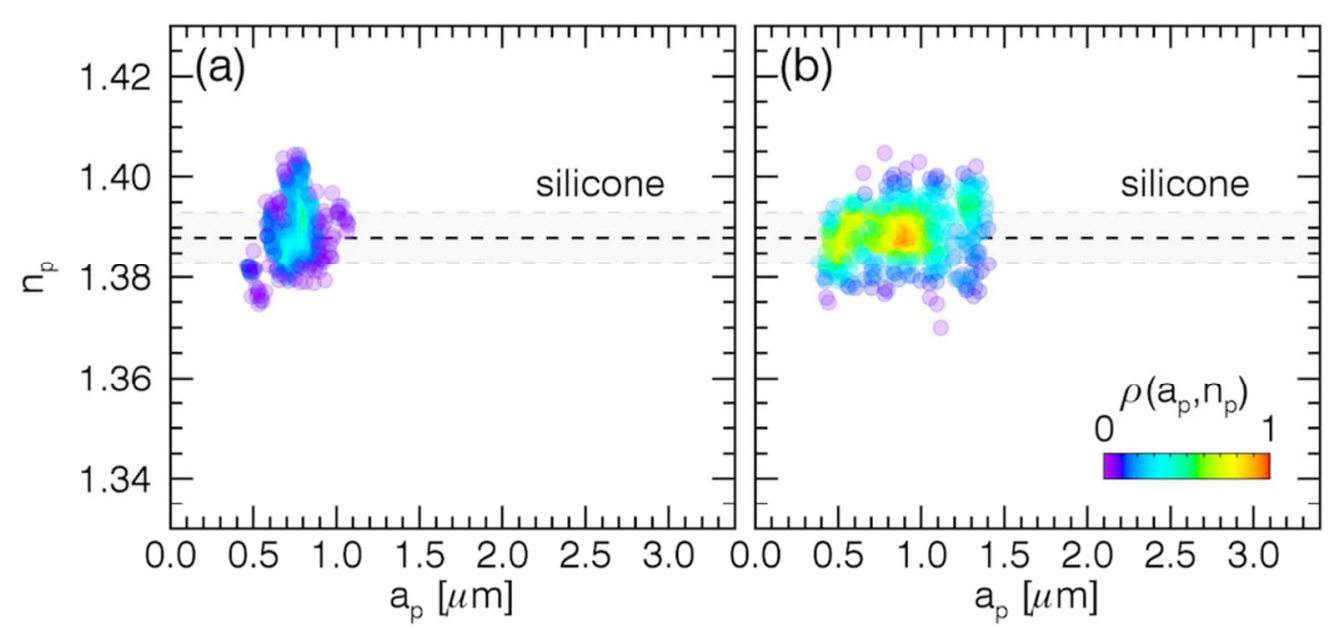

$84 \times 39 \mathrm{~mm}(300 \times 300$ DPI $)$

John Wiley \& Sons, Inc. 


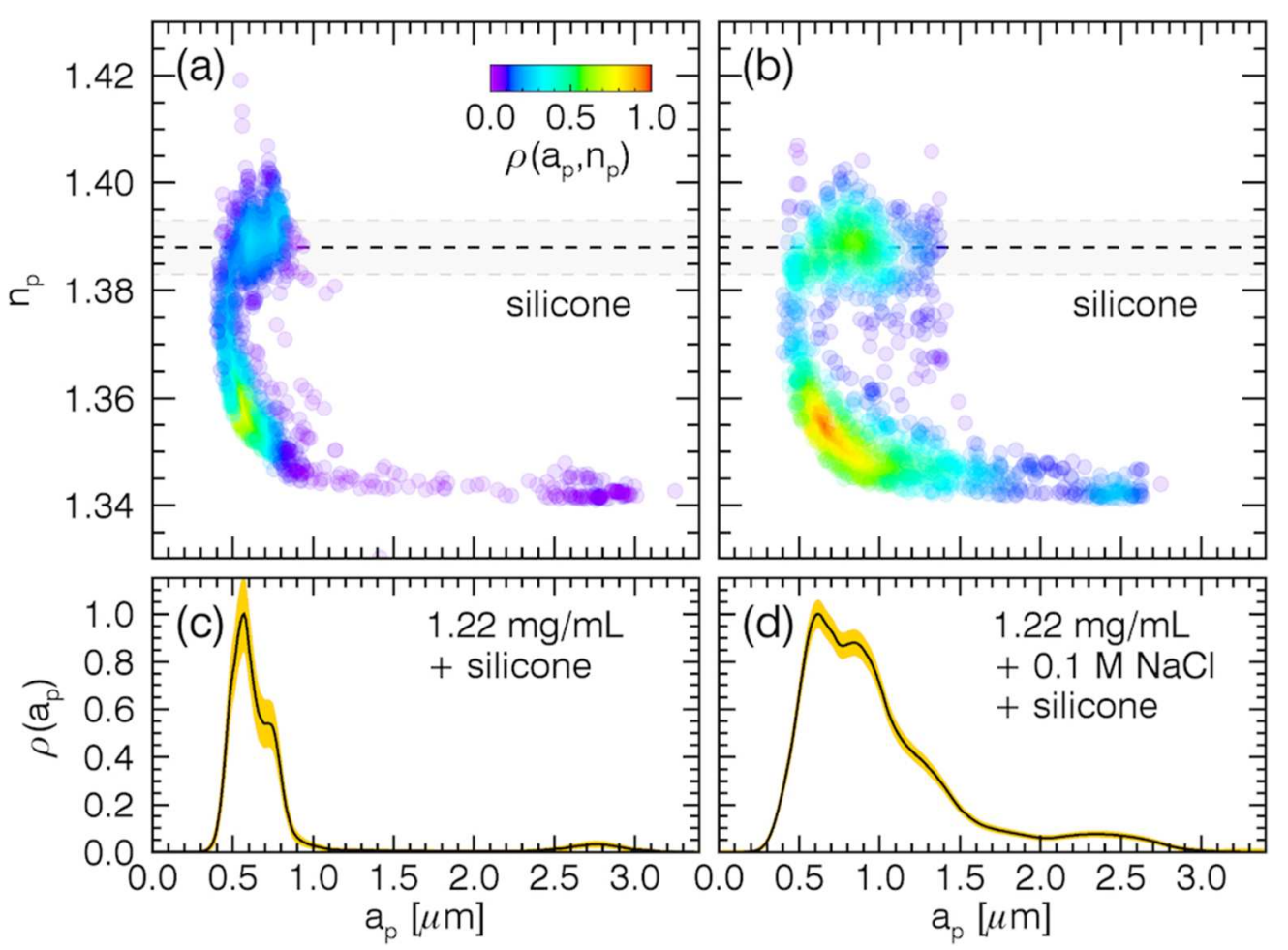

$84 \times 62 \mathrm{~mm}(300 \times 300$ DPI $)$

John Wiley \& Sons, Inc. 